\title{
OPTIMAL CONTROL OF OBSTACLE PROBLEMS: EXISTENCE OF LAGRANGE MULTIPLIERS
}

\author{
MaÏtine Bergounioux ${ }^{1}$ And Fulbert Mignot $^{2}$
}

\begin{abstract}
We study first order optimality systems for the control of a system governed by a variational inequality and deal with Lagrange multipliers: is it possible to associate to each pointwise constraint a multiplier to get a "good" optimality system? We give positive and negative answers for the finite and infinite dimensional cases. These results are compared with the previous ones got by penalization or differentiation.

Résumé. On étudie le problème des systèmes d'optimalité du premier ordre pour le contrôle des systèmes gouvernés par une inéquation variationnelle, et qui prennent en compte les multiplicateurs de Lagrange que l'on peut associer à chaque contrainte ponctuelle. Le problème est étudié en dimension finie puis infinie : suivant les cas des réponses positives ou négatives sont données. Ces résultats sont comparés à ceux obtenus par pénalisation ou différentiation.
\end{abstract}

AMS Subject Classification. 49J40, 49K20, 49K35.

Received March 30, 1999. Revised November 9, 1999.

\section{INTRODUCTION}

We consider an optimal control problem where the state function is the solution to a variational inequality of obstacle type. It is known that this state function may be equivalently defined by a variational equality coupled with pointwise constraints (and integral constraints as well for the infinite dimensional case).

In this paper we focus on the following question: is it possible to associate to these pointwise constraints some Lagrange multipliers which satisfy (together with the optimal state) a (first order) qualified optimality system? A related question is to compare such a system (if it exists) to the ones that have been obtained by many authors with various methods, such as for example penalization or (weak) differentiation.

Let us briefly describe the main different techniques that have been considered to obtain optimality systems:

- approximation of the state-inequation by a penalized equation which solution $y_{\varepsilon}(v)$ is a differentiable function of the control $v$; techniques that are used are of Moreau-Yosida type. One may obtain a penalized adjoint state $p_{\varepsilon}$ and partially pass to the limit in the optimality system defining $\left(y_{\varepsilon}\left(u_{\varepsilon}\right), u_{\varepsilon}, p_{\varepsilon}\left(u_{\varepsilon}\right)\right)\left(u_{\varepsilon}\right.$ being the optimal solution to the penalized problem); one can refer to Barbu [2], Ito and Kunisch [13].

Keywords and phrases: Variational inequalities, optimal control, Lagrange multiplier, obstacle problem.

${ }^{1}$ Département de Mathématiques, UMR 6628, Université d'Orléans, BP. 6759, 45067 Orléans Cedex 2, France;

e-mail: Maitine.Bergounioux@labomath.univ-orleans.fr

${ }^{2}$ Laboratoire de Mathématique, bâtiment 425, Université Paris-Sud, 91405 Orsay, France;

e-mail: Fulbert.Mignot@math.u-psud.fr 
Lagrange multipliers are exhibited and the optimality system is not far from the (qualified) Lagrange system (as defined in Sect. 2). We shall precisely compare these two systems in the sequel.

- Relaxation of the complementarity bilinear condition associated to the obstacle constraint $(y, \xi)_{n}=0$ to get $(y, \xi)_{n} \leq \alpha$ and classical penalization of the different constraints (Bergounioux [3,4]). One obtains a Lagrange system for the approximated problem and related Lagrange multipliers that can be used numerically. However it is not possible to get a lot of informations for the limit problem (when $\alpha \rightarrow 0$ ).

- Use of convex duality (Bergounioux and Dietrich [6]): define a Moreau-Yosida regularized function that involves the Variational Inequality and reformulate the optimal control as a minimization problem of the cost functional with a $\mathcal{C}^{1}$ (non convex) equality constraint. Results are close to the ones of Wenbin and Rubio [20]. An optimality system exists under weak assumptions for a large class of (nonlinear) obstacle problems but the existence of "classical" Lagrange multpliers is not ensured.

- Study of the differentiability properties of the functional $v \mapsto y(v)$ (solution to (2.1)) (Mignot [16]). The conical differentiablity of this mapping allows to derive a general optimality system but does not imply existence of Lagrange multipliers.

- Introduction of a generalized cost functional that takes into account the constraints given by the complementarity conditions (2.2) and use of admissible tests functions (Bermudez and Saguez [10], Mignot and Puel $[17,18])$. Here, an optimality system is obtained with Lagrange multipliers and an adjoint equation "living" on the critical cone at the optimal solution. We shall prove that this system is equivalent to the Lagrange system in the finite dimensional case but not in the infinite dimensional case (we give a counter-example).

- Use of a Lagrangian function and first order conditions to get a saddle-point $(\bar{y}, u, \bar{\xi}, p)$ (Bermudez and Saguez [9]). In this paper, the authors obtain a non qualified optimality system (similar to Kuhn-KaruschTucker system) with Lagrange multipliers. Nevertheless, they let the question of qualification open. When the space dimension is finite, we exhibit a class of systems for which the answer is positive and we provide a counter-example in a more general case. When the space dimension is infinite, a counter example shows that the qualification condition is quite hard to ensure.

Let us mention, at last the recent work of Adams et al. [1] about optimal control of the obstacle. In this paper the authors consider a different problem: indeed, they consider the obstacle function as the control and look for the optimal obstacle with respect of a given cost functional.

The paper is organized as follows: we first describe the problem and give the main results; we first consider a finite dimensional problem (which corresponds to the discretization of a continuous one for example): we are able to give positive or negative answers depending on the control space. In Section 3, we consider the infinite dimensional case and we prove that the so-called Lagrange system always implies the known optimality systems. However, there is no equivalence and we give a counter-example. The last sections are devoted to the proofs of these results.

\section{Finite dimensional Case}

\subsection{Problem statement}

We consider an optimal control problem where the state function is solution to the elliptic obstacle problem:

$$
\left\{\begin{array}{l}
y \in K=\left\{\varphi, \varphi \in \mathbb{R}^{n} ; \varphi_{i} \geq 0, i=1, \ldots, n\right\} \\
a(y, \varphi-y) \geq(f+B v, \varphi-y)_{n} \quad \forall \varphi \in K
\end{array}\right.
$$

- Here $(\cdot, \cdot)_{n}$ is the canonical scalar product in $\mathbb{R}^{n}$, and $|\cdot|_{n}$ will denote the euclidian norm in $\mathbb{R}^{n}$.

- $a$ is a coercive, bilinear form defined on $\mathbb{R}^{n} \times \mathbb{R}^{n}$, associated to a $n \times n$ matrix $A$.

- The control function $v$ belongs to $\mathcal{U}=\mathbb{R}^{m}, B$ is a linear operator: $B \in \mathcal{L}\left(\mathbb{R}^{m}, \mathbb{R}^{n}\right)$ and $\left.f \in \mathbb{R}^{n}\right)$.

- $y \geq 0$ is understood componentwise: $y_{i} \geq 0, i=1, \ldots, n$; we shall denote

$$
\{y \geq 0\}=\left\{i \in\{1, \ldots, n\} \mid y_{i} \geq 0\right\} \text { and }\{y=0\}=\left\{i \in\{1, \ldots, n\} \mid y_{i}=0\right\} .
$$


Inequality (2.1) has a unique solution that we note $y(v)$; moreover it is equivalent to the following system (complementarity conditions)

$$
\left\{\begin{array}{l}
(y, v, \xi) \in \mathbb{R}^{n} \times \mathbb{R}^{m} \times \mathbb{R}^{n} \\
A y-(f+B v)-\xi=0 \\
(y, \xi)_{n}=0 \\
y \geq 0, \xi \geq 0
\end{array}\right.
$$

The cost functional to be minimized is a classical one

$$
J(y(v), v)=\frac{1}{2}\left|C y(v)-z_{d}\right|_{\ell}^{2}+\frac{N}{2}|v|_{m}^{2},
$$

where $\left.C \in \mathcal{L}\left(\mathbb{R}^{n}, \mathbb{R}^{\ell}\right), z_{d} \in \mathbb{R}^{\ell}, N>0\right)$.

Remark 2.1. We may replace the canonical scalar product in $\mathbb{R}^{n}$ by any equivalent scalar product defined for example by

$$
(y, z)_{L, n}=(L y, z)_{n}
$$

where $L$ is a symmetric, positive and definite $M$-matrix. We recall that a $M$-matrix is a non singular matrix, such that all the diagonal elements are positive, the non-diagonal elements are non-positive and the elements of the inverse matrix are nonegative (see [12] for instance). We may also replace $|v|_{m}^{2}$ by $\left(L_{m} v, v\right)_{m}$ where $L_{m}$ is a symmetric, positive and definite $M$-matrix as well; such a term appears if we think of a discretization of the $L^{2}$-norm with a Finite Element method. If the discretization is done with a Finite Difference method then $L_{m}$ may be chosen as the identity matrix. Nevertheless, we keep the above setting (using the canonical scalar product) to make the presentation more clear.

It is well known that the following optimal control

$$
J(y(u), u)=\min _{v \in \mathbb{R}^{m}} J(y(v), v) .
$$

has at least a solution $u$.

First we define the Lagrangian function which plays an important role for our analysis.

We associate to the complementarity conditions (2.2) the application $G$ and the cone $\mathcal{K}$ ( $K$ is the positive cone of $\mathbb{R}^{n}$ ):

$$
\begin{aligned}
& G: \mathbb{R}^{n} \times \mathbb{R}^{m} \times \mathbb{R}^{n} \rightarrow \mathbb{R}^{n} \times \mathbb{R} \times \mathbb{R}^{n} \times \mathbb{R}^{n} \\
&(y, v, \xi) \mapsto\left(A y-(f+B v)-\xi,(y, \xi)_{n}, y, \xi\right), \\
& \mathcal{K}=\{0\} \times\{0\} \times K \times K \\
&(y(v), v, \xi) \text { solution to }(2.2) \Longleftrightarrow(y, v, \xi) \in G^{-1}(\mathcal{K}) .
\end{aligned}
$$

Define the polar cone of $K: K^{o}=\{\zeta, \forall y \in K,(\zeta, y) \leq 0\}(=-K)$. Then

$$
\mathcal{K}^{o}=\mathbb{R}^{n} \times \mathbb{R} \times(-K) \times(-K) .
$$

The Lagrangian function $\mathcal{L}$ is defined as follows

$$
\begin{gathered}
(y, v, \xi) \in \mathbb{R}^{n} \times \mathbb{R}^{m} \times \mathbb{R}^{n} \\
q=\left(q_{1}, q_{2}, q_{3}, q_{4}\right) \in-\mathcal{K}^{o}=\mathbb{R}^{n} \times \mathbb{R} \times K \times K \\
\mathcal{L}(y, v, \xi, q)=J(y, v)-\left(q_{1}, A y-(f+B v)-\xi\right)_{n}-q_{2}(y, \xi)_{n}-\left(q_{3}, y\right)_{n}-\left(q_{4}, \xi\right)_{n}
\end{gathered}
$$


that is, with the natural inner product of the constraints space (denoted $((\cdot, \cdot))$

$$
\begin{gathered}
\mathcal{L}(y, v, \xi, q)=J(y, v)+((-q, G(y, v, \xi))) \\
(y, v, \xi) \in \mathbb{R}^{n} \times \mathbb{R}^{m} \times \mathbb{R}^{n},-q \in \mathcal{K}^{o} .
\end{gathered}
$$

If the lagrangian function $\mathcal{L}$ has a saddle point $(\bar{y}, u, \bar{\xi}, p)$,

$$
\begin{gathered}
\forall(y, v, \xi) \in \mathbb{R}^{n} \times \mathbb{R}^{m} \times \mathbb{R}^{n}, \forall q \in \mathbb{R}^{n} \times \mathbb{R} \times K \times K\left(=-\mathcal{K}^{o}\right), \\
\mathcal{L}(y, v, \xi, p) \geq \mathcal{L}(\bar{y}, u, \bar{\xi}, p) \geq \mathcal{L}(\bar{y}, u, \bar{\xi}, q),
\end{gathered}
$$

then the following (necessary) first order conditions are satisfied

$$
\begin{gathered}
\frac{\partial \mathcal{L}(\bar{y}, u, \bar{\xi}, p)}{\partial(y, v, \xi)}=0, \\
\frac{\partial \mathcal{L}}{\partial q}(\bar{y}, u, \bar{\xi}, p)(q-p) \leq 0, \forall q,-q \in \mathcal{K}^{o} .
\end{gathered}
$$

Our goal is to study the system $(2.9 \mathrm{a}, 2.9 \mathrm{~b})$ that we detail here after.

Relation (2.9b). The function $\mathcal{L}$ is affine with respect to $q$ and the cone $\mathcal{K}$ is convex so that relation $(2.9 \mathrm{~b})$ can written as

$$
\left\{\begin{array}{l}
((G(\bar{y}, u, \bar{\xi}),-q+p)) \leq 0, \quad \forall q,-q \in \mathcal{K}^{o} \\
-p \in \mathcal{K}^{o}
\end{array}\right.
$$

that is

$$
\begin{gathered}
G(\bar{y}, u, \bar{\xi}) \in \mathcal{K}, \\
-p \in \mathcal{K}^{o} \cap(\mathbb{R} G(\bar{y}, u, \bar{\xi}))^{o} .
\end{gathered}
$$

Relation (2.9a) yields

$$
D J(\bar{y}, u, \bar{\xi})-p \circ D G(\bar{y}, u, \bar{\xi})=0,
$$

that is

$$
D J(\bar{y}, u, \bar{\xi})=(D G(\bar{y}, u, \bar{\xi}))^{*}(p) .
$$

Therefore the first order necessary conditions associated to a saddle-point of $\mathcal{L}$ are given by the three relations (2.10).

Remark 2.2 (Geometrical interpretation). Relation (2.10a) means that $\bar{y}=y(u)$ is the solution to the variational inequality with a right-hand side control function $u$.

Relation (2.10b) gives a property for the Lagrange multiplier $p$ :

$$
-p \in\left(\mathcal{S}_{G(\bar{y}, u, \bar{\xi})}(\mathcal{K})\right)^{o}=\mathcal{K}^{o} \cap(\mathbb{R} G(\bar{y}, u, \bar{\xi}))^{\perp},
$$

(The cone which vertex is $G(\bar{y}, u, \bar{\xi})$ and based on $\mathcal{K}$ translated to 0 is denoted $\mathcal{S}_{G(\bar{y}, u, \bar{\xi})}(\mathcal{K})$.)

At last, equation (2.10c) makes the relation between the gradient of the cost functional and the Lagrange multiplier precise. 
Since the dimension space is finite, we may detail furthermore. Using the vectors components, relations (2.10) lead to

$$
\begin{gathered}
(\bar{y}, u, \bar{\xi}) \in \mathbb{R}^{n} \times \mathbb{R}^{m} \times \mathbb{R}^{n} \\
p=\left(p_{1}, p_{2}, p_{3}, p_{4}\right) \in \mathbb{R}^{n} \times \mathbb{R} \times \mathbb{R}^{n} \times \mathbb{R}^{n} .
\end{gathered}
$$

- State inequation

$$
\begin{array}{ll}
A \bar{y}-(f+B u)-\bar{\xi}=0 & \frac{\partial \mathcal{L}}{\partial q_{1}}=0 \\
\bar{y} \geq 0 & \frac{\partial \mathcal{L}}{\partial q_{3}} \leq 0 \\
\bar{\xi} \geq 0 & \frac{\partial \mathcal{L}}{\partial q_{4}} \leq 0 \\
(\bar{y}, \bar{\xi})_{n}=0 & \frac{\partial \mathcal{L}}{\partial q_{2}}=0 .
\end{array}
$$

- Multipliers constraints

$$
\begin{gathered}
\begin{cases}p_{3} \geq 0 & -p \in \mathcal{K}^{o} \\
\left(p_{3}, \bar{y}\right)_{n}=0 & \left(\frac{\partial \mathcal{L}}{\partial q_{3}}, p_{3}\right)=0\end{cases} \\
\begin{cases}p_{4} \geq 0 & -p \in \mathcal{K}^{o} \\
\left(p_{4}, \bar{\xi}\right)_{n}=0 & \left(\frac{\partial \mathcal{L}}{\partial q_{4}}, p_{4}\right)=0 .\end{cases}
\end{gathered}
$$

- Adjoint system

$$
\begin{array}{ll}
A^{*} p_{1}+p_{2} \bar{\xi}+p_{3}=C^{*}\left(C \bar{y}-z_{d}\right) & \frac{\partial \mathcal{L}}{\partial y}=0 . \\
B^{*} p_{1}=-N u & \frac{\partial \mathcal{L}}{\partial v}=0 \\
p_{1}-p_{2} \bar{y}-p_{4}=0 & \frac{\partial \mathcal{L}}{\partial \xi}=0 .
\end{array}
$$

$A^{*}$ (respectively $B^{*}, C^{*}$ ) denotes the adjoint operator (matrix) of $A$ (respectively $B, C$ ). We have written on the right hand side, the meaning as Lagrangian derivatives at $(\bar{y}, u, \bar{\xi}, p))$.

Now we want to study the system $(2.11-2.13)$ that we call Lagrange system associated to the control problem (2.1-2.4). 
Remark 2.3. In this paper we decided to focus on the unilateral obstacle problem. Of course we could consider many other variational inequalities as for example the one corresponding to

$$
K=\{z \in V|| \nabla z(x) \mid \leq 1, \text { a.e. in } \Omega\} .
$$

Our purpose is to review and compare different optimality systems and we have decided to choose a "simple" framework. We have not studied this case and the analysis is certainly less easy.

\subsection{Main results}

\subsubsection{A counter-example}

Without any modification of the Lagrange system or without any additional assumption on the data, the existence of Lagrange multipliers for the optimal control problem we consider is not ensured. As a counterexample we consider the following: the control space is one dimensional, the state-space is $\mathbb{R}^{3}$ and the matrix $A$ is the matrix obtained with the discretization of the Laplacian operator $-\frac{d^{2}}{d x^{2}}$ on $[0,1]$ (Dirichlet conditions and 3-points discretization). We shall give details for this example in Section 5.

\subsubsection{Comparison with optimality systems}

We compare here the Lagrange system to the optimality system that have been obtained by Mignot [16] and Mignot and Puel $[17,18]$.

The (conical) differentiability properties of the state with respect to the control (Mignot [16] or the use of a generalized cost functional (Mignot and Puel $[17,18]$ ) allow to get a useful optimality system (for many situations) which includes the systems obtained by penalization. We first prove here (Th. 2.4) that the system obtained in $[17,18]$ is equivalent to the Lagrange system (for the finite dimensional case) that is, sets of solutions are the same. The precise result is given hereafter.

Theorem 2.4. The following assertions are equivalent:

i) The optimality system $(\mathcal{O S})_{1}:(2.1,2.14,2.15)$

$$
\begin{gathered}
\left\{\begin{array}{l}
a(\bar{y}, \varphi-\bar{y}) \geq(f+B u, \varphi-\bar{y})_{n}, \forall \varphi \in K \\
\bar{y} \in K
\end{array}\right. \\
\left\{\begin{array}{l}
\left.a(\varphi, p) \leq C^{*}\left(C \bar{y}-z_{d}\right), \varphi\right)_{n}, \quad \forall \varphi \in S_{\bar{y}} \\
p \in S_{\bar{y}} \stackrel{\text { def }}{=}\left\{\varphi, \varphi \mid\{\bar{y}=0\} \geq 0,(\varphi, \bar{\xi})_{n}=0\right\}
\end{array}\right. \\
B^{*} p+N u=0
\end{gathered}
$$

has a solution $(\bar{y}, u, p) \in \mathbb{R}^{n} \times \mathbb{R}^{m} \times \mathbb{R}^{n}$.

ii) The Lagrange system (2.11-2.13) has a solution $\left(\bar{y}, u, \bar{\xi}, p_{1}, p_{2}, p_{3}, p_{4}\right) \in \mathbb{R}^{n} \times \mathbb{R}^{m} \times \mathbb{R}^{n} \times \mathbb{R}^{n} \times \mathbb{R} \times \mathbb{R}^{n} \times \mathbb{R}^{n}$.

One switches from the solution to the Lagrange system (2.11, 2.12, 2.13) to the solution to optimality system (2.1, 2.14, 2.15) keeping $\bar{y}$ and $u$ and taking $p=p_{1}$.

Taking the previous counter-example into account, this equivalence result shows that the conical differentiability of the state with respect to the control is not a sufficient condition to get the existence of a solution to the optimality system $(\mathcal{O S})_{1}$. Let us recall criterion given in Mignot [16] to ensure the existence: if $u$ is an optimal control (the control space is denoted $\mathcal{U}$ ) then

$$
D y(u)(\mathcal{U}) \subset A^{-1}(B(\mathcal{U}))
$$


is a sufficient condition. This assumption has the major inconvenient (as qualification conditions) to deal with a property of the optimal solution which is of course a priori unknown. The following result is a partial answer to this problem.

Thanks to the previous analysis, if the control space is $\mathbb{R}^{n}$ and the state is defined by

$$
\left\{\begin{array}{l}
a(y(v), \varphi-y(v)) \geq(f+v, \varphi-y(v))_{n}, \forall \varphi \in K \\
y(v) \in K,
\end{array}\right.
$$

the optimality system $(\mathcal{O S})_{1}$ has a solution (Mignot and Puel [17]) and it is equivalent to the Lagrange system (2.11-2.13) with Theorem 2.4. Therefore, we are able to set a modified Lagrange system which allows to consider many other situations. More precisely, we consider a problem where

- the control space is $\mathbb{R}^{m} \subset \mathbb{R}^{n}$ :

$$
\mathbb{R}^{m}=\left\{\left(v_{i}\right)_{i=1, \ldots, n}, v_{m+1}=\ldots=v_{n}=0\right\},
$$

- the state function is solution to (2.16),

- the bilinear form $a$ of (2.16) is associated to a $M$-matrix $A$;

$$
a(\varphi, \psi)=(A \varphi, \psi)=\sum_{i, j=1}^{n} a_{i j} \varphi_{j} \psi_{i}
$$

- the cost functional is given by $(2.3)$.

We get the following result

Theorem 2.5. Consider the optimal control problem defined above such that $f$ satisfies

$$
\forall i=m+1, \ldots, n, \quad f_{i} \geq 0 .
$$

If $u$ is an optimal control and if we denote $\bar{y}=\left(\bar{y}^{1}, \bar{y}^{2}\right) \in \mathbb{R}^{m} \times \mathbb{R}^{n-m}, \bar{\xi}=\left(\bar{\xi}^{1}, \bar{\xi}^{2}\right) \in \mathbb{R}^{m} \times \mathbb{R}^{n-m}$, there exists $\left(p_{1}, p_{2}, p_{3}, p_{4}\right) \in \mathbb{R}^{n} \times \mathbb{R} \times \mathbb{R}^{m} \times \mathbb{R}^{m}$ solution to

$$
\begin{gathered}
\left\{\begin{array}{c}
A \bar{y}-f-B u-\bar{\xi}=0 \\
\bar{y} \geq 0, \bar{\xi} \geq 0,(\bar{y}, \bar{\xi})_{n}=0 .
\end{array}\right. \\
p_{3} \geq 0 \quad\left(p_{3}, \bar{y}^{1}\right)_{m}=0, \\
p_{4} \geq 0 \quad\left(p_{4}, \bar{\xi}^{1}\right)_{m}=0 . \\
A^{*} p_{1}+p_{2} \bar{\xi}+B p_{3}=C^{*}\left(C \bar{y}-z_{d}\right), \\
B^{*} p_{1}=-N u \\
B^{*} p_{1}-p_{2} \bar{y}^{1}-p_{4}=0
\end{gathered}
$$

where $B$ is the canonical injection from $\mathbb{R}^{m}$ to $\mathbb{R}^{n}$. 
Remark 2.6. This "modified" Lagrange system is different from the previous one (2.11-2.13) since multipliers $p_{4}$ and $p_{3}$ belong to $\mathbb{R}^{m}$ (and no longer to $\mathbb{R}^{n}$ ) and relation (2.21c) lives in $\mathbb{R}^{m}$.

Remark 2.7. This result will allow to treat the approximated problem for the control of unilateral problems in $H_{o}^{1}(\Omega)$ with $L^{2}(\omega)$, as control space, $\omega \in \Omega$ and $f \geq 0$ on $(\Omega \backslash \omega)$. We detail with Example 3.3.1.

\subsubsection{Comparison to optimality systems obtained via a penalization method}

Now, we focus on the comparison of the Lagrange system to the optimality system that has been obtained by Ito and Kunisch $[13,14]$.

Noting the equivalence between

$$
\xi=\max (0, \xi-c y),(c>0)
$$

and the complementarity conditions

$$
y \geq 0, \xi \geq 0,(y, \xi)_{n}=0,
$$

Ito and Kunisch [14] have obtained an optimality system (with a penalization method) for the case of unilateral control problems with smooth data; this system precises the one obtained before by Barbu [2]. The adaptation of the Ito and Kunisch system to the finite dimensional case (with the previous notations) ascertains that the following system $(\mathcal{O S})_{2}$ has (at least) a solution corresponding to the optimal state

$$
\begin{gathered}
(\bar{y}, u, \bar{\xi}, p, \mu) \in \mathbb{R}^{n} \times \mathbb{R}^{m} \times \mathbb{R}^{n} \times \mathbb{R}^{n} \times \mathbb{R}^{n} \\
\left\{\begin{array}{l}
A \bar{y}=f+B u+\bar{\xi} \\
\bar{y} \geq 0, \bar{\xi} \geq 0,(\bar{y}, \bar{\xi})_{n}=0, \\
A^{*} p+\mu=C^{*}\left(C \bar{y}-z_{d}\right), \\
\mu_{i} \bar{y}_{i}=0, \quad i=1, \ldots, n, \\
p_{i} \bar{\xi}_{i}=0, \quad i=1, \ldots, n, \\
(p, \mu)_{n}=0, \\
B^{*} p=-N u .
\end{array}\right.
\end{gathered}
$$

Considering the Lagrange system $(2.11-2.13)$ we recover $(\mathcal{O S})_{2}$, setting $p=p_{1}$ and $\mu=p_{2} \bar{\xi}+p_{3}$. The converse does not work because the system $(\mathcal{O S})_{2}$ does not let appear the respective contributions of the three constraints $\left.\bar{y} \geq 0, \bar{\xi} \geq 0,(\bar{y}, \bar{\xi})_{n}=0\right)$, but we must emphasize that no assumption on the operator $B$ nor $f$ is required to get a solution to $(\mathcal{O S})_{2}$; therefore, this may be used for more general situations than the one we study here.

Moreover, if the cost functional is

$$
J(y, v)=\frac{1}{2}\left|y-z_{d}\right|_{n}^{2}+\frac{N}{2}|v|_{m}^{2}
$$

if $B$ is the canonical injection from $\mathbb{R}^{m}$ to $\mathbb{R}^{n}$, and $z_{d} \leq 0$ then $(\mathcal{O S})_{2}$ is sufficient as well: its solution corresponds to the optimum $u$.

Unfortunately, without the condition $z_{d} \leq 0$, it is not always the case; the following control problem:

- state equation: $A=I_{n}, B v=(\alpha v, \beta v)$ and $f \equiv 0$;

- control: $v \in \mathbb{R}$;

- state: $y(v) \in \mathbb{R}^{2}, y(v)=\left(\alpha v^{+}, \beta v^{+}\right),(\alpha \geq 0, \beta \geq 0)$;

- $\operatorname{cost} J(y, v)=\frac{1}{2}\left|y-z_{d}\right|_{2}^{2}+\frac{1}{2} v^{2}$

admits $u=0$ as an optimal solution if and only if

$$
\alpha z_{d}^{1}+\beta z_{d}^{2} \leq 0 .
$$

For any $\left(z_{d}^{1}, z_{d}^{2}\right)$, system $(\mathcal{O S})_{2}$ has always the solution $u=0, y=\xi=0, p=(0,0), \mu=-z_{d}$, which does not correspond to the optimum if $\alpha z_{d}^{1}+\beta z_{d}^{2}>0$. (For $u=0$, the corresponding Lagrange system (2.11-2.13) has a solution only if $z_{d}^{1} \leq 0 \quad z_{d}^{2} \leq 0$.) 


\section{INFINITE DIMENSIONAL CASE}

\subsection{Problem statement}

We consider a simple model case (that can be easily generalized to any continuous, $H_{o}^{1}$-elliptic bilinear form $a)$. The variational inequality defining the state function is the "continuous" version of (2.1).

$$
\left\{\begin{array}{l}
y(v) \in K=\left\{\varphi \mid \varphi \in H_{o}^{1}(\Omega), \varphi \geq 0 \text { a.e. }\right\}, \\
\forall \varphi \in K \quad \int_{\Omega}\left(\nabla y(v), \nabla(\varphi-y(v)) d x \geq \int_{\Omega}(f+B v)(\varphi-y(v)) d x,\right.
\end{array}\right.
$$

where

- $\Omega$ is a open domain of $\mathbb{R}^{k}(k \leq 3)$ with a smooth boundary $\partial \Omega$.

- $(\cdot, \cdot)_{\Omega}$ denotes the $L^{2}(\Omega)$-inner product and $\|\cdot\|_{\Omega}$ the $L^{2}(\Omega)$-norm.

- The control space is $\mathcal{U} \subset L^{2}(\Omega)$ and $B \in \mathcal{L}\left(\mathcal{U}, L^{2}(\Omega)\right)$.

- $f \in L^{2}(\Omega)$.

Inequality (3.1) may be written in the "complementarity" form

$$
\left\{\begin{array}{l}
-\Delta y(v)-(f+B v)-\xi(v)=0 \\
y(v) \geq 0, \xi(v) \geq 0 \\
(y(v), \xi(v))_{\Omega}=0
\end{array}\right.
$$

All inequalities ( $y \geq 0$ for instance) are understood almost everywhere. The cost function is defined similarly to $(2.3)$ :

$$
J(y(v), v)=\frac{1}{2}\left\|C y(v)-z_{d}\right\|_{\Omega}^{2}+\frac{N}{2}\|v\|_{\Omega}^{2}
$$

where $C$ is a linear continuous operator from $H_{o}^{1}(\Omega)$ to $L^{2}(\Omega)$ and $z_{d} \in L^{2}(\Omega)$.

It is well known that the optimal control problem

$$
J(y(u), u)=\min _{v \in \mathcal{U}} J(y(v), v)
$$

has at least a solution $u$ (see [2] for example). As in the finite dimensional case we may define the Lagrangian function $\mathcal{L}$

$$
\begin{gathered}
(y, v, \xi) \in H_{o}^{1}(\Omega) \times \mathcal{U} \times L^{2}(\Omega) \\
q=\left(q_{1}, q_{2}, q_{3}, q_{4}\right) \in H_{o}^{1}(\Omega) \times \mathbb{R} \times L^{2}(\Omega) \times L^{2}(\Omega) \\
q_{3} \geq 0, q_{4} \geq 0, \\
\mathcal{L}(y, v, \xi, q)=J(y, v)-\left\langle q_{1},-\Delta y-(f+v)-\xi\right\rangle-q_{2}(y, \xi)_{\Omega}-\left(q_{3}, y\right)_{\Omega}-\left(q_{4}, \xi\right)_{\Omega}
\end{gathered}
$$

where $\langle\cdot, \cdot\rangle$ denotes the duality product between $H_{o}^{1}(\Omega)$ and $H^{-1}(\Omega)$.

\subsection{Main results}

The optimal control $u$ being given, we may consider a Lagrange system analogous to (2.11-2.13); for convenience we write this (continuous) system:

$$
\begin{gathered}
(\bar{y}, u, \bar{\xi}) \in H_{o}^{1}(\Omega) \times L^{2}(\Omega) \times L^{2}(\Omega) \\
p=\left(p_{1}, p_{2}, p_{3}, p_{4}\right) \in L^{2}(\Omega) \times \mathbb{R} \times L^{2}(\Omega) \times L^{2}(\Omega) .
\end{gathered}
$$




$$
\left.\begin{array}{l}
p_{3} \geq 0,\left(p_{3}, \bar{y}\right)_{\Omega}=0 \\
p_{4} \geq 0,\left(p_{4}, \bar{\xi}\right)_{\Omega}=0
\end{array}\right\} \quad \text { Multipliers constraints }
$$

$$
\left.\begin{array}{l}
A^{*} p_{1}+p_{2} \bar{\xi}+p_{3}=C^{*}\left(C \bar{y}-z_{d}\right), \\
B^{*} p_{1}=-N u, \\
p_{1}-p_{2} \bar{y}-p_{4}=0,
\end{array}\right\} \text { Adjoint system }
$$

$A^{*}$ (respectively $B^{*}, C^{*}$ ) denotes the adjoint operator of $A$ (respectively $B, C$ ).

One checks if system (3.6) has a solution or not. In view of the finite dimensional case, we may give only a positive elementary result concerning its relations to already known optimality systems.

Proposition 3.1. If $(\bar{y}, u, \bar{\xi}),\left(p_{1}, p_{2}, p_{3}, p_{4}\right)$, is a solution to the (continuous) Lagrange system (3.6) then the classical optimality system (still denoted $\left.(\mathcal{O S})_{1}\right)$ that can be obtained with the differentiability method or the generalized cost functional, admits $\left(\bar{y}, p_{1}=p\right)$ as a solution:

$$
\begin{gathered}
a(\bar{y}, \varphi-\bar{y}) \geq(f+B u, \varphi-\bar{y})_{\Omega}, \forall \varphi \in K, \quad \bar{y} \in K \\
p \in S_{\bar{y}}=\left\{\varphi, \varphi \in H_{o}^{1}(\Omega), \varphi_{\mid\{\bar{y}=0\}} \geq 0 \quad \text { q.e. } ;(\varphi, \bar{\xi})_{\Omega}=0\right\}, \\
a(\varphi, p) \leq\left(C^{*}\left(C \bar{y}-z_{d}\right), \varphi\right)_{\Omega}, \forall \varphi \in S_{\bar{y}} \\
B^{*} p+N u=0 .
\end{gathered}
$$

(q.e. means quasi everywhere in the sense of the capacity of $H_{o}^{1}(\Omega)$ ).

The converse implication is false: this means that we cannot split the adjoint state $p$ of $(\mathcal{O S})_{1}$ in a part corresponding to the constraint $(y, \xi)_{\Omega}=0$ and a part corresponding to the constraint $\xi \geq 0$ ( for the finite dimensional case we had $\left.p=p_{2} \bar{y}+p_{4}\right)$. Let us present a counter-example. The state $y(v)$ is the solution to the variational inequality (where $f(x)=-x$ ):

$$
\begin{aligned}
& y(v) \in K=\left\{\varphi, \varphi \in H_{o}^{1}(0,1) \mid \varphi(x) \geq 0, \forall x \in[0,1]\right\}, \\
& \int_{0}^{1} y^{\prime}\left(\varphi^{\prime}-y^{\prime}\right) d x \geq \int_{0}^{1}(-x+v)(\varphi-y) d x
\end{aligned}
$$

and the cost functional turns to be

$$
J(y, v)=\frac{1}{2} \int_{0}^{1}(y-\varepsilon)^{2} d x+\frac{1}{2} \int_{0}^{1} v^{2} d x
$$

$\left(z_{d}=\varepsilon\right.$, where $\varepsilon$ is such that $\left.0<\varepsilon<\frac{\sqrt{3}}{16} \pi^{2}\right)$.

The corresponding Lagrange system has no solution while the optimality system $(\mathcal{O S})_{1}$ gets one. 
Remark 3.2. Note that the previous example satisfies a strict complementarity criterion, that is the measure of the set

$$
\{x \in \Omega \mid \bar{y}(x)=0 \text { and } \bar{\xi}(x)=0\}
$$

is null. This example let us think that we have no "classical" assumption to propose (strict-complementarity for example) to ensure the existence of solutions to the Lagrange system (in a qualified form). A Lagrange system in a non qualified form has been obtained in [9] (or [8] for a more general problem) and Lagrange multipliers are measures.

In addition, the previous example shows that even if the optimal control $u$ is a Frechet - differentiability point of the state-application: $u \mapsto y(u)$ it may happens that the Lagrange system has no solution, though there may exists a adjoint state. More precisely the cone $S_{\bar{y}}$ defined by $(3.7 \mathrm{~b})$ is here a closed subspace and the optimality system is $(3.7 \mathrm{a}, 3.7 \mathrm{~b}, 3.7 \mathrm{~d})$ and

$$
a(\varphi, p)=\left(C^{*}\left(C \bar{y}-z_{d}\right), \varphi\right)_{\Omega} \quad \forall \varphi \in S_{\bar{y}} .
$$

This shows that the Lagrange system is quite restrictive.

Nevertheless, analogously to finite dimensional results one can enounce an existence theorem.

Proposition 3.3. Let $\bar{y}, p$ the solution to $(\mathcal{O S})_{1}$ and set $q=C^{*}\left(C y-z_{d}\right)-A^{*} p$.

Assume there exists $m \in \mathbb{R}$ such that

$$
q(x) \geq m \bar{\xi}(x) \quad p(x) \geq m \bar{y}(x) \text { a.e. on }\{x \in \Omega \mid \bar{y}(x)=0\} .
$$

Then, the Lagrange system (3.5-3.6) has a solution.

The proof is the same as in the finite dimensionnal case. Nevertheless, this result is not useful since assumptions deal with the optimal solution which is unknown of course...

\subsection{Approximated problems}

The previous results allow to solve discretized versions of some control problems.

\subsubsection{Example 1.}

The state $y(v)$ is defined as the solution to the variational inequality

$$
\begin{aligned}
& y(v) \in K=\left\{\varphi, \varphi \in H_{o}^{1}(\Omega) \mid \varphi(x) \geq 0, \forall x \in \Omega\right\}, \\
& \int_{\Omega}\left(\nabla y(v), \nabla(\varphi-y(v)) d x \geq \int_{\Omega}\left(f+v 1_{\omega}\right)(\varphi-y(v)) d x .\right.
\end{aligned}
$$

The control space is $L^{2}(\omega),(\omega \subset \Omega$ with a positive measure); it is assumed that $f \geq 0$ on $\Omega \backslash \omega$. The indicatrix funtion of $\omega\left(1_{\omega}(x)=1\right.$ if $x \in \omega$ and 0 if not) is denoted $1_{\omega}$. The cost functional is classical (see (2.3))

$$
J(y, v)=\frac{1}{2}\left\|C y-z_{d}\right\|_{\Omega}^{2}+\frac{1}{2}\|v\|_{\omega}^{2} .
$$

Consider an approximating sequence (in n-finite dimensional spaces) of the state inequation:

$$
\left\{\begin{array}{l}
y_{h} \in K_{h}=\left\{\varphi \in V_{h}, \varphi \geq 0\right\} \\
\forall \varphi \in K_{h} \quad a_{h}\left(y_{h}, \varphi-y_{h}\right) \geq \int_{\Omega}\left(f_{h}+v_{h} 1_{\omega}\right)\left(\varphi-y_{h}\right) d x
\end{array}\right.
$$

where $h=h(n)$, such that the matrix $A_{h}$ discretizing the operator is a $M$ - matrix. One can use, for example, a FEM method which provides a stiffness $M$-matrix (for instance (for the $2 \mathrm{D}$ case), it is sufficient to use a 
2D-triangulation $\mathcal{T}_{h}$ where any triangle angle is less that $\pi / 2$ (Ciarlet and Raviart [11]). In this case, we have to consider a scalar product in $\mathbb{R}^{n}$ adapted to the discretization and make minor modifications to the cost functional (to take into account the discretization of the $L^{2}$-norm) as mentionned in Remark 2.1.

It is also possible to use a finite difference method: the matrix discretizing the Laplacian operator is known to be a $M$-matrix.

We suppose in addition that the discretization (sub)-space corresponding to the (sub)-triangulation of $\omega$ is of dimension $m$ (that is we have $m V_{h}$-basis functions "living" on $\omega$ ). The approximate solution $u_{h}$ of the (finite dimensional) control problem

$$
\mathcal{P}_{h} \quad \min _{v_{h} \in \mathbb{R}^{m}} J\left(y_{h}, v_{h}\right)
$$

converges to the solution to the continuous problem (see $[5,7,19]$ for instance). Theorem 2.5 may be used for problem $\mathcal{P}_{h}$ : there exists an associated Lagrange system. When the parameter $h$ tends towards 0 we get the convergence of $\left(y_{h}, u_{h}, p_{1 h}\right)$ to $(y, u, p)$ solution to the continuous problem optimality system, but there may be no convergence for the multiplier $p_{2, h}$; this multiplier corresponds to the nonlinear constraint, $(y, \xi)_{\Omega}=0$ which have a degenerate behavior generating many difficulties.

\subsubsection{Example 2}

We get an approximation result, similar to the one we obtained for Example 1; this concerns control problems governed by a Signorini type variational inequality.

$$
\begin{gathered}
y \in K=\left\{\varphi, \varphi_{\mid \Gamma} \geq 0\right\},(\Gamma \subset \partial \Omega), \\
\forall \varphi \in K \quad \int_{\Omega}(\nabla y, \nabla(\varphi-y)) d x=\int_{\Omega} f(\varphi-y) d x+\int_{\Gamma} v(\varphi-y) d \Gamma
\end{gathered}
$$

and $f \geq 0$ over $\Omega$.

\section{PROOFS}

\subsection{Finite dimensional case}

\subsubsection{Counter example}

We detail the counter example presented in Section (2.2.1): we exhibit a control problem where the Lagrange system (2.11-2.13) has no solution.

The state space is $\mathbb{R}^{3}$ (with the usual inner product) while the control space is $\mathbb{R}$. The bilinear form $a$ is associated to the (symmetric) matrix $A$ :

$$
\begin{gathered}
y=\left(\begin{array}{l}
y_{1} \\
y_{2} \\
y_{3}
\end{array}\right), A=\left(\begin{array}{ccc}
2 & -1 & 0 \\
-1 & 2 & -1 \\
0 & -1 & 2
\end{array}\right) \quad\left(=A^{*}\right) \\
a(y, z)=(A y, z),(y, z) \in \mathbb{R}^{3} \times \mathbb{R}^{3} .
\end{gathered}
$$

The positive cone of $\mathbb{R}^{3}$ is still denoted $K$ and the operator $B$ is: $v \mapsto(0, v, 0)$. The state of the system $y(v)=\left(y_{i}(v)\right), i=1,2,3$, is solution to

$$
(A y, \varphi-y) \geq(-f)\left(\varphi_{1}-y_{1}\right)+(2 f+v)\left(\varphi_{2}-y_{2}\right)+(-f)\left(\varphi_{3}-y_{3}\right), \forall \varphi \in K, y \in K,
$$


and may be written with the multiplier $\xi \in \mathbb{R}^{3}$ as

$$
\left\{\begin{array}{c}
2 y_{1}-y_{2}=-f+\xi_{1} \\
-y_{1}+2 y_{2}-y_{3}=2 f+v+\xi_{2} \\
-y_{2}+2 y_{3}=-f+\xi_{3} \\
y_{i} \geq 0, \xi_{i} \geq 0, y_{i} \xi_{i}=0, i=1,2,3
\end{array}\right.
$$

with $f \in \mathbb{R}, f>0$. The solution $y(v)$ can be explicitly computed:

Case $v \geq 0$

$$
\left\{\begin{array}{lll}
y_{1}=\frac{v}{2} & , & \xi_{1}=0 \\
y_{2}=f+v & , & \xi_{2}=0 \\
y_{3}=\frac{v}{2} & , & \xi_{3}=0
\end{array}\right.
$$

Case $-2 f \leq v \leq 0$

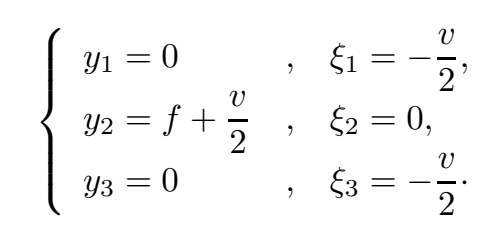

Case $v \leq-2 f$

$$
\begin{cases}y_{1}=0 \quad, \quad \xi_{1}=f \\ y_{2}=0 \quad, \quad \xi_{2}=-(2 f+v) \\ y_{3}=0 \quad, \quad \xi_{3}=f\end{cases}
$$

The cost functional is

$$
J(y, v)=\frac{1}{2} \sum_{i=1}^{3}\left(y_{i}-z_{i}\right)^{2}+\frac{N}{2} v^{2},(N>0) .
$$

Data $f$ and $z$ must verify

$$
\begin{gathered}
f>0, \\
2\left(f-z_{2}\right)-\left(z_{1}+z_{3}\right) \geq 0, \\
\left(f-z_{2}\right) \leq 0 .
\end{gathered}
$$

Proposition 4.1. With assumptions (4.5), we get

$$
\min _{v \in \mathbb{R}} J(y(v), v)=J(y(0), 0),
$$

(and the optimal control is unique: $u=0$ ). 
Proof. - If $v>0$ (taking (4.3a) into account),

$$
J(y(v), v)=\frac{1}{2}\left[\left(\frac{v}{2}-z_{1}\right)^{2}+\left(f+v-z_{2}\right)^{2}+\left(\frac{v}{2}-z_{3}\right)^{2}\right]+\frac{N}{2} v^{2}
$$

that is

$$
J(y(v), v)=\frac{1}{2}\left[2\left(f-z_{2}\right)-\left(z_{1}+z_{3}\right)\right] v+\frac{1}{4}(3+2 N) v^{2}+J(y(0), 0)
$$

and, with (4.5b)

$$
\forall v>0 \quad J(y(v), v)>J(y(0), 0) .
$$

- If $-2 f \leq v \leq 0$, we have similarly

$$
\begin{gathered}
J(y(v), v)=\frac{1}{2}\left[\left(-z_{1}\right)^{2}+\left(f+\frac{v}{2}-z_{2}\right)^{2}-\left(-z_{3}\right)^{2}\right]+\frac{N}{2} v^{2}, \\
J(y(v), v)=\frac{1}{2}\left[\left(f-z_{2}\right) v+\left(N+\frac{1}{4}\right) v^{2}\right]+J(y(0), 0)
\end{gathered}
$$

and, with $(4.5 \mathrm{c})$

$$
J(y(v), v) \geq J(y(0), 0) .
$$

- If $v \leq-2 f$

$$
J(y(v), v)=J(y(0,0))+f\left(z_{2}-f\right)+\frac{N}{2} v^{2} \geq J(y(0), 0) .
$$

The Lagrange system $(2.11-2.13)$ associated to the optimal control $u=0, \bar{y}(u)=(0, f, 0), \bar{\xi}=(0,0,0)$ reads

$$
\begin{gathered}
p_{1} \in \mathbb{R}^{3}, \quad p_{2} \in \mathbb{R}, \quad p_{3} \in \mathbb{R}^{3}, \quad p_{4} \in \mathbb{R}^{3}, \\
p_{1}=\left(p_{1}^{i}\right)_{i=1,2,3}, \quad p_{3}=\left(p_{3}^{i}\right)_{i=1,2,3}, \quad p_{4}=\left(p_{4}^{i}\right)_{i=1,2,3}
\end{gathered}
$$

State inequation

$$
\begin{gathered}
p_{3} \geq 0, \quad\left(p_{3}, \bar{y}\right)=f p_{3}^{2}=0, \\
p_{4} \geq 0, \quad\left(p_{4}, \bar{\xi}\right)=\left(p_{4}, 0\right)=0 .
\end{gathered}
$$

$$
\begin{gathered}
A p_{1}+p_{3}=(\bar{y}-z), \\
B^{*} p_{1}=N u=0 \\
p_{1}-p_{2} \bar{y}-p_{4}=0 .
\end{gathered}
$$

Study of (4.6-4.7): Relations (4.6a-4.6b) imply

$$
p_{3}^{1} \geq 0, p_{3}^{2}=0, p_{3}^{3} \geq 0
$$




$$
p_{4}^{1} \geq 0, p_{4}^{2} \geq 0, p_{4}^{3} \geq 0
$$

and $(4.7 \mathrm{~b})$ :

$$
p_{1}^{2}=0
$$

With the three components of (4.7c) we obtain

$$
\begin{gathered}
p_{1}^{1}=p_{4}^{1} \geq 0, \\
-p_{2} f=p_{4}^{2} \geq 0, \\
p_{1}^{3}=p_{4}^{3} \geq 0 .
\end{gathered}
$$

It remains (4.7a) (a three equations system)

$$
\left\{\begin{array}{l}
2 p_{1}^{1}-p_{1}^{2}+p_{3}^{1}=\bar{y}_{1}-z_{1} \\
-p_{1}^{1}+2 p_{1}^{2}-p_{1}^{3}+p_{3}^{2}=\bar{y}_{2}-z_{2} \\
-p_{1}^{2}+2 p_{1}^{3}+p_{3}^{3}=\bar{y}_{3}-z_{3} .
\end{array}\right.
$$

As $\bar{y}_{1}=\bar{y}_{3}=0, \bar{y}_{2}=f, p_{1}^{2}=0=p_{3}^{2}$, this gives

$$
\begin{gathered}
2 p_{1}^{1}+p_{3}^{1}=-z_{1} \\
-p_{1}^{1}-p_{1}^{3}=f-z_{2} \\
2 p_{1}^{3}+p_{3}^{3}=-z_{3} .
\end{gathered}
$$

With (4.8a) and (4.9), equations (4.10a) and (4.10c) require

$$
z_{1} \leq 0, \quad z_{3} \leq 0 .
$$

On the other hand such conditions are not needed by conditions $(4.5 \mathrm{a}-4.5 \mathrm{~b})$ (which assert that the unique optimal control is $u=0$, for example, $f=1, z_{2}=2, z_{1}=-4, z_{3}=1$ ).

Remark 4.2. 1. If the Lagrange system gets a solution, then conditions (4.5a) and (4.5b) are satisfied, and the solution is an optimum.

2. If $z_{1} \leq 0, z_{3} \leq 0$, then the Lagrange system gets a solution.

Remark 4.3. Usually, for finite dimensional cases, looking for Lagrange multipliers associated to

$$
\min _{G(v) \in \mathcal{K}} J(v)=J(u)
$$

(where functions are smooth enough and $\mathcal{K}=\{0\} \times\left(\mathbb{R}^{+}\right)^{q}$ ), needs three steps:

1. Passage to first order

$$
\begin{gathered}
D J(u)(w) \geq 0, \\
\forall w \in \mathcal{S}_{u}\left(G^{-1}(\mathcal{K})\right)=\left\{w, \exists v_{n}, G\left(v_{n}\right) \in \mathcal{K}, \lim v_{n}=u,\right. \\
\left.\exists \lambda_{n} \rightarrow \infty, w=\lim \lambda_{n}\left(v_{n}-u\right)\right\} .
\end{gathered}
$$


Condition $(\beta)$ can be written

$$
-D J(u) \in\left(\mathcal{S}_{u}\left(G^{-1}(\mathcal{K})\right)\right)^{o} .
$$

2. Linearization of the cone $\mathcal{S}_{u}\left(G^{-1}(\mathcal{K})\right)$. First,

$$
\mathcal{S}_{u}\left(G^{-1}(\mathcal{K})\right) \subset(D G(u))^{-1}\left(\mathcal{S}_{G(u)}(\mathcal{K})\right)
$$

is always true and we have to establish equality $(\gamma)$

$$
\text { convex hull of } \left.\mathcal{S}_{u}\left(G^{-1}(\mathcal{K})\right)\right)=\left(D G(u)^{-1}\left(\mathcal{S}_{G(u)}(\mathcal{K})\right)\right.
$$

This step corresponds to Kuhn and Tucker sufficient qualification conditions without nonlinear equality constraints.

3. Farkas Minkowski lemma

With this lemma (the cones we consider here are polyhedral for the finite dimensional case) we get

$$
\left((D G(u))^{-1}\left(\mathcal{S}_{G(u)}(\mathcal{K})\right)\right)^{o}=(D G(u))^{*}\left(\mathcal{S}_{G(u)}(\mathcal{K})\right)^{o} .
$$

Therefore, if equation $(\gamma)$ is ensured, then we get the multiplier, that is

$$
D J(u) \in(D G(u))^{*}\left(\mathcal{S}_{G(u)}(\mathcal{K})\right)^{o} .
$$

Here, Step 2 fails: we may have a strict inclusion in relation $(\gamma)$ (Kurcyusz [15], Kurcyusz and Zowe [21]). Let us detail: always considering the previous counter-example, a simple computation gives

$$
\begin{gathered}
\mathcal{S}_{G(u)}(\mathcal{K})=\left(\begin{array}{l}
0 \\
0 \\
0
\end{array}\right) \times\{0\} \times\left(\begin{array}{l}
\mathbb{R}^{+} \\
\mathbb{R} \\
\mathbb{R}^{+}
\end{array}\right) \times\left(\begin{array}{l}
\mathbb{R}^{+} \\
\mathbb{R}^{+} \\
\mathbb{R}^{+}
\end{array}\right) \text {and } \\
D G(u)^{-1}\left(\mathcal{S}_{G(u)}(\mathcal{K})\right)=\left\{\left[\left(\begin{array}{l}
y_{1} \\
y_{2} \\
y_{3}
\end{array}\right), v,\left(\begin{array}{l}
\xi_{1} \\
\xi_{2} \\
\xi_{3}
\end{array}\right)\right] \text { such that }\left\{\begin{array}{l}
y_{1}, y_{2}, \xi_{1}, \xi_{2}, \xi_{3} \geq 0 \\
2 y_{1}-y_{2}-\xi_{1}=0 \\
-y_{1}+2 y_{2}-y_{3}-v=0 \\
-y_{2}+2 y_{3}-\xi_{3}=0
\end{array}\right\} .\right.
\end{gathered}
$$

Another (longer) computation shows that

$$
\mathcal{S}_{u}\left(G^{-1}(\mathcal{K})\right) \subset \mathcal{S}=\left\{(y, v, \xi) \mid \xi_{2}=0, A y-v-\xi=0\right\} \subset\left\{(y, v, \xi) \mid \xi_{1}=\xi_{3}\right\}
$$

Therefore, to get the strict inclusion in relation $(\gamma)$, it is sufficient to exhibit an element of $D G(u)^{-1}\left(\mathcal{S}_{G(u)}(\mathcal{K})\right.$ such that $\xi_{1} \neq \xi_{3}$. We may choose for example $y=(1,0,2), v=-3, \xi=(2,0,4)$.

\subsubsection{Proof of Theorem 2.4}

From Optimality system $(\mathcal{O S})_{1}$ to Lagrange system

Inequation (2.14) reads (with $\left.q=C^{*}\left(C \bar{y}-z_{d}\right)-A^{*} p\right)$

$$
(q, \varphi)_{n} \geq 0 \forall \varphi \in S_{\bar{y}}
$$

that is

$$
\begin{gathered}
q=0 \text { on }\{\bar{y}>0\}, \\
q \geq 0 \text { on }\{\bar{y}=0\} \backslash\{\bar{\xi}>0\},
\end{gathered}
$$


(no condition for $(\bar{\xi}>0)$ ).

We set $p_{1}=p$; to get a solution to $(2.12,2.13)$ we have to find $p_{2}, p_{3}, p_{4}$ such that

$$
\begin{gathered}
q=p_{2} \bar{\xi}+p_{3}, \\
p_{3} \geq 0, \quad\left(p_{3}, \bar{y}\right)_{n}=0,
\end{gathered}
$$

and

$$
p=p_{2} \bar{y}+p_{4}, p_{4} \geq 0,\left(p_{4}, \bar{\xi}\right)_{n}=0 .
$$

Let us notice first, that for any $p_{2}$, relations $\left(q-p_{2} \bar{\xi}, \bar{y}\right)_{n}=\left(p-p_{2} \bar{y}, \bar{\xi}\right)_{n}=0$ are true since $(p, \bar{\xi})_{n}=0=(q, \bar{y})_{n}$. Therefore, we have to choose $p_{2}$ negative "enough" to get

$$
q-p_{2} \bar{\xi} \geq 0
$$

and

$$
p-p_{2} \bar{y} \geq 0
$$

\section{Relation (4.15)}

- on $\{\bar{y}>0\}, q=\bar{\xi}=0$ so $q-p_{2} \bar{\xi}=0$,

- on $\{\bar{y}=0\} \cap\{\bar{\xi}=0\}$ (with (4.12b)) $q \geq 0$,

- on $\{\bar{\xi}>0\}(\subset\{\bar{y}=0\})$, we must have $q_{i}>p_{2} \bar{\xi}_{i}$, so

$$
\min _{i \in\{\bar{\xi}>0\}}\left(\frac{q_{i}}{\bar{\xi}_{i}}\right) \geq p_{2}
$$

\section{Relation (4.16)}

- on $\{\bar{y}=0\}, p-p_{2} \bar{y}=p \geq 0$ (since $p \in S_{\bar{y}}$ ),

- on $\{\bar{y}>0\}$ we must have $p_{i} \geq p_{2} \bar{y}_{i}$, therefore

$$
\min _{i \in\{\bar{y}>0\}}\left(\frac{p_{i}}{\bar{y}_{i}}\right) \geq p_{2}
$$

It is sufficient to choose

$$
p_{2}=\min \left(\min _{\left(\bar{\xi}_{i}>0\right)}\left(\frac{q_{i}}{\bar{\xi}_{i}}\right), \min _{\left(\bar{y}_{i}>0\right)}\left(\frac{p_{i}}{\bar{y}_{i}}\right)\right)
$$

to get a solution to the Lagrange system.

\section{Conversely: From Lagrange system to Optimality system $(\mathcal{O S})_{1}$}

If $\left(\bar{y}, u, \bar{\xi}, p_{1}, p_{2}, p_{3}, p_{4}\right)$ is a solution to the Lagrange system then $\left(\bar{y}, u, \bar{\xi}, p=p_{1}\right)$ is solution to $(\mathcal{O S})_{1}$. Indeed, let be $\varphi \in S_{\bar{y}}$, and use (2.13a): we get

$$
a\left(\varphi, p_{1}\right)=\left(C^{*}\left(C \bar{y}-z_{d}\right), \varphi\right)_{n}-p_{2}(\bar{\xi}, \varphi)_{n}-\left(p_{3}, \varphi\right)_{n} .
$$

First, $(\varphi, \bar{\xi})_{n}=0$ because $\varphi \in S_{\bar{y}}$ and $\left(p_{3}, \varphi\right)_{n} \geq 0\left(p_{3}=0\right.$ on $\{\bar{y}>0\}, p_{3} \geq 0$ on $\left.\{\bar{y}=0\}\right)$. Therefore

$$
a\left(\varphi, p_{1}\right) \leq\left(C^{*}\left(C \bar{y}-z_{d}\right), \varphi\right)_{n}, \quad \forall \varphi \in S_{\bar{y}} .
$$


We must verify that $p_{1} \in \mathcal{S}_{\bar{y}}$. With $(2.13 \mathrm{c}), p_{1}=p_{2} \bar{y}+p_{4}$, so $p_{1} \geq 0$ on $\{\bar{y}=0\}$ since $p_{4} \geq 0$ and $\left(p_{1}, \bar{\xi}\right)_{n}=p_{2}(\bar{y}, \bar{\xi})_{n}+\left(p_{4}, \bar{\xi}\right)_{n}=0$.

The triple $\left(u, \bar{y}, p_{1}\right)$ is solution to the optimality system $(\mathcal{O S})_{1}$.

\subsubsection{Proof of Theorem 2.5}

We show first that the original problem turns to be an equivalent control problem on $\mathbb{R}^{m}$ (instead of $\mathbb{R}^{n}$ ) to which Mignot and Puel [17] results may be applied. Then it remains to prove that the Lagrange system we obtain in $\mathbb{R}^{m}$ may be written as in Theorem 2.5 (relations $(2.20)$ and (2.21)).

The following lemma gives a precise form for the complementarity conditions defining the state $y(v)$.

Lemma 4.4. Let $A$ be a $M$-matrix and $y$ the solution to the variational inequality (associated to $g \in \mathbb{R}^{n}$ ), written as following

If $k$ is such that $g_{k} \geq 0$, then $\xi_{k}=0$.

$$
\left\{\begin{array}{l}
A y=g+\xi \\
y \geq 0, \quad \xi \geq 0, \quad(y, \xi)=0
\end{array}\right.
$$

Proof. The line number $k$ of $A y=g+\xi$ can be written

$$
a_{k k} y_{k}+\sum_{j \neq k} a_{k j} y_{j}=g_{k}+\xi_{k}
$$

If $\xi_{k}>0$ then $y_{k}=0$ and with $a_{k j} \leq 0 \quad j \neq k$, we get a contradiction:

$$
0 \geq \sum_{j \neq k} a_{k j} y_{j} \geq g_{k}+\xi_{k}>0
$$

With assumptions on the control space $\mathcal{U} \mathcal{U}=\mathbb{R}^{m}$ and $f(2.18)$, the state $y(v)$ and the multiplier $\xi(v)$ are such that

$$
\begin{gathered}
(A y(v))_{i}=f_{i}+v_{i}+\xi_{i}(v), \quad i=1, \ldots, m, \\
(A y(v))_{i}=f_{i}, \quad i=m+1, \ldots, n, \\
y(v) \geq 0 \\
\xi(v) \geq 0 \\
\quad i=m+1, \ldots, n \\
\xi_{i}(v)=0 \quad \\
(y(v), \xi(v))_{n}=0 .
\end{gathered}
$$

Moreover, we have

Lemma 4.5. The optimal control $u$ satisfies: $\xi_{k}(u)>0 \Longrightarrow u_{k}=0$. 
Proof. Let be $k$ such that $\xi_{k}(u)>0$. With $(4.22 \mathrm{c}, 4.22 \mathrm{~d})$ and $(4.22 \mathrm{f})$, this yields $y_{k}(u)=0$ and $(4.22 \mathrm{e})$ gives $0 \leq k \leq m$. Let us call $e_{k}$ the $k$-th vector of the canonical basis of $\mathbb{R}^{n}$. With uniqueness of the solution to the variational inequality we remark that

$$
y(u)=y\left(u+t e_{k}\right), \forall t, \quad 0<t \leq \xi_{k}(u)
$$

indeed the multiplier associated to the control $\left(u+t e_{k}\right)$ is $\left(\xi(u)-t e_{k}\right)$ and the pair $\left.\left(y\left(u+t e_{k}\right), \xi(u)-t e_{k}\right)\right)$ satisfies the same complementarity conditions as $(y(u), \xi(u))$. Then, we get

$$
\begin{gathered}
J(y(u), u) \leq J\left(y\left(u+t e_{k}\right), u+t e_{k}\right)=J\left(y(u), u+t e_{k}\right), \\
0 \leq \frac{d}{d t} J\left(y(u), u+t e_{k}\right)_{\mid t=0}=N u_{k} \\
0 \leq u_{k} .
\end{gathered}
$$

Assume $u_{k}>0$; then

$$
y\left(u_{1}, \ldots, u_{k-1}, u_{k}, u_{k+1}, \ldots, u_{m}\right)=y\left(u_{1}, \ldots, u_{k-1}, 0, u_{k}, \ldots, u_{m}\right)
$$

since for $\tilde{u}=u-u_{k} e_{k}$ one sets $y(\tilde{u})=y(u)$ and $\xi(\tilde{u})=\xi(u)+u_{k} e_{k}$. Same complementarity conditions are once again satisfied for the pairs $(y(\tilde{u}), \xi(\tilde{u}))$ and $(y(u), \xi u))$. Thus,

$$
J(y(\tilde{u}), \tilde{u})=J\left(y(u), u-u_{k} e_{k}\right)=J(y(u), u)-\frac{N}{2}\left(u_{k}\right)^{2}<J(y(u), u)
$$

and $u_{k}=0$.

Remark 4.6. Lemma (4.5) does not need $M$-matrices.

The inequation defining the state $y=\left(y^{1}, y^{2}\right) \in \mathbb{R}^{m} \times \mathbb{R}^{n-m}$ can be split: the matrix $A$ is written with blocks,

$$
A=\left(\begin{array}{cc}
A_{11} & A_{12} \\
A_{21} & A_{22}
\end{array}\right), \quad A_{11} \in \mathcal{M}_{m, m}(\mathbb{R}), \quad A_{12} \in \mathcal{M}_{m, n-m}(\mathbb{R}) \ldots
$$

where $\mathcal{M}_{m, n}(\mathbb{R})$ denotes the set of real $m \times n$ matrices.

Relation (4.22b) becomes $A_{21} y^{1}+A_{22} y^{2}=f^{2}$, that is

$$
y^{2}=\left(A_{22}\right)^{-1}\left(-A_{21} y^{1}+f^{2}\right)
$$

and the first component $y^{1}$ is solution to:

$$
\left\{\begin{array}{c}
\left(A_{11}-A_{12} A_{22}^{-1} A_{21}\right) y^{1}=f^{1}+v-A_{12} A_{22}^{-1} f^{2}+\xi^{1}, \\
y^{1} \geq 0, \xi^{1} \geq 0,\left(y^{1}, \xi^{1}\right)_{p}=0 .
\end{array}\right.
$$

As $\left(A_{21}-A_{12} A_{22}^{-1} A_{21}\right)$ is definite positive, the first component $y^{1}$ is solution to a variational inequality in $\mathbb{R}^{m}$, with a control function living in $\mathbb{R}^{m}$ : then we know that the optimal control satisfies an optimality system (Mignot [16], Mignot and Puel [17]).

The cost functional may be written with respect to $y^{1}$ as well $\left(C=\left(C^{1}, C^{2}\right)\right)$.

$$
\left(C^{1} \in \mathcal{M}_{\ell, m}(\mathbb{R}), \quad C^{2} \in \mathcal{M}_{\ell, n-m}(\mathbb{R})\right)
$$




$$
\begin{aligned}
J(y, v) & =\frac{1}{2}\left|C^{1} y^{1}+C^{2} y^{2}-z_{d}\right|_{\ell}^{2}+\frac{N}{2}|v|_{m}^{2} \\
& =\frac{1}{2}\left|C^{1} y^{1}+C^{2}\left(A_{22}\right)^{-1}\left(-A_{21} y^{1}+f^{2}\right)-z_{d}\right|_{\ell}^{2}+\frac{N}{2}|v|_{m}^{2} \\
& =\frac{1}{2}\left|\left(C^{1}-C^{2}\left(A_{22}\right)^{-1} A_{21}\right) y^{1}-\left(z_{d}-C^{2}\left(A_{22}\right)^{-1} f^{2}\right)\right|_{\ell}^{2}+\frac{N}{2}|v|_{m}^{2} .
\end{aligned}
$$

The Lagrange system associated to an optimal pair $\left(u, \bar{y}=\left(\bar{y}^{1}, \bar{y}^{2}\right)\right)$ is:

There exists $\left(q_{1}, q_{2}, q_{3}, q_{4}\right) \in \mathbb{R}^{m} \times \mathbb{R} \times \mathbb{R}^{m} \times \mathbb{R}^{m}$

such that

$$
\begin{gathered}
q_{3} \geq 0, \quad\left(q_{3}, \bar{y}^{1}\right)_{m}=0, \\
q_{4} \geq 0, \quad\left(q_{4}, \bar{\xi}^{1}\right)_{m}=0, \quad\left(\bar{\xi}^{1}=\bar{\xi}\right) . \\
\left(A_{11}^{*}-A_{21}^{*}\left(A_{22}^{*}\right)^{-1} A_{12}^{*}\right) q_{1}+q_{2} \bar{\xi}+q_{3} \\
=\left(C^{1}-C^{2}\left(A_{22}\right)^{-1} A_{21}\right)^{*}\left[\left(C^{1}-C^{2}\left(A_{22}\right)^{-1} A_{21}\right) \bar{y}^{1}-\left(z_{d}-C^{2}\left(A_{22}\right)^{-1} f^{2}\right)\right] \\
q_{1}=-N u, \\
q_{1}-q_{2} \bar{y}^{1}-q_{4}=0 .
\end{gathered}
$$

The vector $p_{1}=\left(p^{1}, p^{2}\right) \in \mathbb{R}^{m} \times \mathbb{R}^{n-m}$ is then defined by

$$
p^{1}=q^{1}
$$

and

$$
p^{2}=\left(A_{22}^{*}\right)^{-1}\left\{-\left(A_{12}\right)^{*} q_{1}+\left(C^{2}\right)^{*}\left[C^{1} \bar{y}^{1}-z_{d}+C^{2}\left(A_{22}\right)^{-1}\left(-A_{21} \bar{y}^{1}+f^{2}\right)\right]\right\},
$$

which implies (with (4.23))

$$
A_{22}^{*} p^{2}+A_{12}^{*} p^{1}=\left(C^{2}\right)^{*}\left[C^{1} \bar{y}^{1}+C^{2} \bar{y}^{2}-z_{d}\right] .
$$

Equation (4.26a) becomes

$$
A_{21}^{*} p^{1}+A_{22}^{*} p^{2}+q_{2} \bar{\xi}+q_{3}=\left(C^{1}\right)^{*}\left[C^{1} \bar{y}^{1}+C^{2} \bar{y}^{2}-z_{d}\right] .
$$

Equations (4.27a) and (4.27b) are the components of

$$
A^{*} p_{1}+p_{2} \bar{\xi}+B p_{3}=C^{*}\left(C \bar{y}-z_{d}\right)
$$

where we have set $p_{2}=q_{2}\left(\in \mathbb{R}^{m}\right)$ ( $B$ injection from $\mathbb{R}^{m}$ to $\left.\mathbb{R}^{n}\right), p_{3}=q_{3} \in \mathbb{R}^{m}$, that is (2.21a).

Equations (4.26b) and (4.26c) are nothing else that (2.21b) and (2.21c).

This achieves the proof. 
Remark 4.7. Here is an example where Theorem 2.5 may be applied (and not Th. 2.4). The matrix $A$ is the same as in counter-example (4.1.1).

$$
A=\left(\begin{array}{ccc}
2 & -1 & 0 \\
-1 & 2 & -1 \\
0 & -1 & 2
\end{array}\right)
$$

The second member $f$ is $\left(\begin{array}{l}0 \\ 0 \\ 0\end{array}\right)$ and the control space is $\{0\} \times \mathbb{R} \times\{0\}$. The state system is

$$
(A y(v), \varphi-y(v)) \geq v\left(\varphi_{2}-y_{2}(v)\right) \quad \forall \varphi \in\left(\left[0, \infty[)^{3}, \quad y(v) \in\left(\left[0, \infty[)^{3},\right.\right.\right.\right.
$$

and the cost functional $J$

$$
J(y, v)=\frac{1}{2} \sum_{i=1}^{3}\left(y_{i}-z_{d, i}\right)^{2}+\frac{1}{2} v^{2}
$$

where $z_{d}$ is such that

$$
z_{1}+2 z_{2}+z_{3}<0
$$

We may verify that $\min _{v} J(y(v), v)=J(y(0), 0)$. Theorem 2.5 may be applied. However, if the Lagrange system defined by Theorem 2.4 has a solution then $z_{i} \leq 0 i=1,2,3$, which are more restrictive conditions than (4.29).

\subsection{Infinite dimensional case}

\subsubsection{Proof of proposition 3.1}

Assume that the Lagrange system (3.6) has a solution $(\bar{y}, u, \bar{\xi}), p=\left(p_{1}, p_{2}, p_{3}, p_{4}\right)$ and let us prove that $\left(\bar{y}, u, p_{1}\right)$ is solution to $(\mathcal{O S})_{1}$.

Relations (3.6a) and (3.7a) are the same; similarly (3.7d) is part of adjoint system (3.6c). First equation of (3.6c) gives

$$
a\left(\varphi, p_{1}\right)=\left(A \varphi, p_{1}\right)_{\Omega}=\left(\varphi, A^{*} p_{1}\right)_{\Omega}=\left(C^{*}\left(C \bar{y}-z_{d}\right), \varphi\right)_{\Omega}-\left(\varphi, p_{2} \bar{\xi}+p_{3}\right)_{\Omega} .
$$

Choose $\varphi \in S_{\bar{y}}$, so that $(\varphi, \bar{\xi})_{\Omega}=0$ and $\varphi \geq 0$ on $\{\bar{y}=0\}$. The first equation of (3.6b) yields that $p_{3}=0$ (a.e) on $\{\bar{y}>0\}$. Therefore

$$
\left(\varphi, p_{3}\right)_{\Omega}=\left(\varphi, p_{3}\right)_{\{\bar{y}=0\}}+\left(\varphi, p_{3}\right)_{\{\bar{y}>0\}}=\left(\varphi, p_{3}\right)_{\{\bar{y} \geq 0\}} \geq 0
$$

and we get (3.7c). It remains to prove that $p_{1} \in S_{\bar{y}}$. We know that $p_{1} \in H_{o}^{1}(\Omega)$ and with the last equation of (3.6c): $p_{1}=p_{2} \bar{y}+p_{4}$; so

- $p_{1}=p_{4} \geq 0$ on $\{\bar{y}=0\}$ (with the second equation of $(3.6 \mathrm{~b})$ ),

- $\left(p_{1}, \bar{\xi}\right)_{\Omega}=p_{2}(\bar{y}, \bar{\xi})_{\Omega}+\left(p_{4}, \bar{\xi}\right)_{\Omega}=0$ with the second equation of $(3.6 \mathrm{~b})$ and the state inequation.

The converse is false and we present a counter-example.

Proposition 4.8. The control problem defined by (3.8) (state) and (3.9) (cost)

$$
\min _{v \in L^{2}(0,1)} J(y(v), v)
$$

admits $u=0, y(u)=\bar{y}=0, \xi(u)=\bar{\xi}=x$ as a solution. 
With this proposition (whose proof is the technical part of the counter-example), we are able to show that the Lagrange system has no solution. This system reads

$$
\begin{gathered}
-\frac{d^{2} p_{1}}{d x^{2}}+p_{2} \xi+p_{3}=\bar{y}-z_{d}=0-\varepsilon=-\varepsilon, \\
-p_{1}=u=0, \\
p_{1}-p_{2} \bar{y}-p_{4}=0 \\
p_{3} \geq 0, \quad\left(p_{3}, \bar{y}\right)=0 \\
p_{4} \geq 0, \quad\left(p_{4}, \bar{\xi}\right)=0
\end{gathered}
$$

$p_{1} \in H_{o}^{1}(0,1), p_{2} \in \mathbb{R}, p_{3} \in L^{2}(0,1), p_{4} \in L^{2}(0,1)$.

With $(4.30 \mathrm{~b})$ we get $p_{1}=0$ and with $(4.30 \mathrm{e})$, since $\bar{\xi}(x)=x>0$, one gets $p_{4}=0$ as well. It remains to verify (4.30a) and (4.30d):

$$
p_{3}=-\left(\varepsilon+p_{2} x\right), p_{3} \geq 0, p_{2} \in \mathbb{R} .
$$

This has no solution since $\left.\lim _{x \rightarrow 0}\left(\varepsilon+p_{2} x\right)=\varepsilon>0\right)$.

\subsubsection{Proof of proposition 4.8}

The inequality

$$
\forall v \in L^{2}(0,1), J(y(v), v) \geq J(y(0), 0)=J(0,0)
$$

is

$$
\frac{1}{2} \int_{0}^{1}(y(v)-\varepsilon)^{2} d x+\frac{1}{2} \int_{0}^{1} v^{2} d x \geq \frac{1}{2} \int_{0}^{1} \varepsilon^{2} d x
$$

that is

$$
\forall v \in L^{2}(0,1), \int_{0}^{1}(y(v))^{2} d x+\int_{0}^{1} v^{2} d x-2 \varepsilon \int_{0}^{1} y(v) d x \geq 0 .
$$

This inequality is obvious if $y(v) \equiv 0$. So, we suppose that $y(v) \not \equiv 0$. To prove (4.31) the idea is the following: though the term $\int_{0}^{1} y(v) d x$ is a first order term ( $\varepsilon$ is fixed) it will be bounded above by the quadratic terms $\int_{0}^{1}(y(v))^{2} d x$ and $\int_{0}^{1} v^{2} d x$ (we are in a neighborhood of 0 ): indeed, to let $y(v) \not \equiv 0$ "somewhere" it is necessary for $v(x)$ to be "greater" than $x$ so that the contribution of $\int v^{2} d x$ will not be negligible.

As $y=y(v) \in H_{o}^{1}(0,1) \cap H^{2}(0,1) \subset \mathcal{C}^{1}([0,1])$,

$$
\begin{gathered}
\left.(y(v))^{-1}(] 0, \infty[)=\bigcup_{n \in \mathbb{N}}\right] a_{n}, b_{n}[, \\
y(v)\left(a_{n}\right)=y(v)\left(b_{n}\right)=0, \\
y^{\prime}(v)\left(a_{n}\right)=y^{\prime}\left(v_{n}\right)\left(b_{n}\right)=0, \\
\forall x \in] a_{n}, b_{n}\left[,-y^{\prime \prime}(v)(x)=f(x)+v(x) .\right.
\end{gathered}
$$


Lemma 4.9. With the previous notations

$$
\forall n, \int_{a_{n}}^{b_{n}} y(v)(x) d x \leq \frac{\left(b_{n}-a_{n}\right)^{5 / 2}}{\pi^{2}}\left(\int_{a_{n}}^{b_{n}} v^{2}(x) d x\right)^{1 / 2}
$$

Proof. We use Poincaré inequality in $H_{o}^{1}(] a_{n}, b_{n}[):\left(\lambda_{1}=\frac{\pi^{2}}{\left(b_{n}-a_{n}\right)^{2}}\right)$.

$$
\forall y \in H_{o}^{1}(] a_{n}, b_{n}\left[, \quad \int_{a_{n}}^{b_{n}} y^{2} d x \leq \frac{\left(b_{n}-a_{n}\right)^{2}}{\pi^{2}} \int_{a_{n}}^{b_{n}}\left(y^{\prime}\right)^{2} d x\right.
$$

On the other hand, as inequality (3.8) turns to be an equation on $] a_{n}, b_{n}[$, we get ( with $y(v)=y$ )

$$
\int_{a_{n}}^{b_{n}}\left(y^{\prime}\right)^{2} d x=\int_{a_{n}}^{b_{n}}(-x+v) y d x
$$

As $y \geq 0 \int_{a_{n}}^{b_{n}} x y d x \geq 0$, and

$$
\int_{a_{n}}^{b_{n}}\left(y^{\prime}\right)^{2} d x \leq \int_{a_{n}}^{b_{n}} v y d x .
$$

Inequalities (4.34) and (4.35) imply

$$
\int_{a_{n}}^{b_{n}} y^{2} d x \leq \frac{\left(b_{n}-a_{n}\right)^{2}}{\pi^{2}} \int_{a_{n}}^{b_{n}} v y d x \leq \frac{\left(b_{n}-a_{n}\right)^{2}}{\pi^{2}}\left(\int_{a_{n}}^{b_{n}} v^{2} d x\right)^{1 / 2}\left(\int_{a_{n}}^{b_{n}} y^{2} d x\right)^{1 / 2}
$$

therefore

$$
\left(\int_{a_{n}}^{b_{n}} y^{2} d x\right)^{1 / 2} \leq \frac{\left(b_{n}-a_{n}\right)^{2}}{\pi^{2}}\left(\int_{a_{n}}^{b_{n}} v^{2} d x\right)^{1 / 2}
$$

together with

$$
\int_{a_{n}}^{b_{n}} y d x \leq\left(b_{n}-a_{n}\right)^{1 / 2}\left(\int_{a_{n}}^{b_{n}}(y)^{2} d x\right)^{1 / 2}
$$

gives estimation (4.36).

Using (4.33) and (4.36) we get

$$
\begin{aligned}
\int_{a_{n}}^{b_{n}} y^{2} d x+\int_{a_{n}}^{b_{n}} v^{2} d x-2 \varepsilon \int_{a_{n}}^{b_{n}} y d x & \geq \int_{a_{n}}^{b_{n}} v^{2} d x-2 \varepsilon \frac{\left(b_{n}-a_{n}\right)^{5 / 2}}{\pi^{2}}\left(\int_{a_{v}}^{b_{n}} v^{2} d x\right)^{1 / 2} \\
& =\left(\int_{a_{n}}^{b_{n}} v^{2} d x\right)^{1 / 2}\left[\left(\int_{a_{n}}^{b_{n}}(v)^{2} d x\right)^{1 / 2}-2 \varepsilon \frac{\left(b_{n}-a_{n}\right)^{5 / 2}}{\pi^{2}}\right]
\end{aligned}
$$


that is finally

$$
\int_{0}^{1} y^{2} d x+\int_{0}^{1} v^{2} d x-2 \varepsilon \int_{0}^{1} y d x \geq \sum_{n}\left(\int_{a_{n}}^{b_{n}} v^{2} d x\right)^{1 / 2}\left[\left(\int_{a_{n}}^{b_{n}}(v)^{2} d x\right)^{1 / 2}-2 \varepsilon \frac{\left(b_{n}-a_{n}\right)^{5 / 2}}{\pi^{2}}\right]
$$

We go back to the previous situation $\left(a_{n}=a, b_{n}=b\right)$.

Lemma 4.10. The function $v$ which appears in the problem

$$
\left\{\begin{array}{l}
\left.-y^{\prime \prime}=-x+v \text { on }\right] a, b[ \\
y(a)=y(b)=0 \\
y(x)>0 \text { on }] a, b[
\end{array}\right.
$$

satisfies

$$
\frac{(b-x)}{(b-a)} \int_{a}^{x}(\sigma-a) v(\sigma) d \sigma+\frac{(x-a)}{(b-a)} \int_{x}^{b}(b-\sigma) v(\sigma) d \sigma \geq \frac{1}{6}(x+(a+b))(x-a)(b-x) .
$$

Proof. Let $\varphi$ be the solution to

$$
\left.-\varphi^{\prime \prime}=v \text { on }\right] a, b[, \varphi(a)=\varphi(b)=0
$$

and $\psi$ the solution to

$$
\left.-\psi^{\prime \prime}=x \text { on }\right] a, b[, \psi(a)=\psi(b)=0 .
$$

As $y(x)=\varphi(x)-\psi(x) \geq 0$, we have

$$
\varphi(x) \geq \psi(x) .
$$

The left hand side term in (4.38) is $\varphi$, the right hand side term is $\psi$.

With (4.37) it is sufficient to prove

$$
\forall n,\left(\int_{a_{n}}^{b_{n}} v^{2} d x\right)^{1 / 2} \geq 2 \varepsilon \frac{\left(b_{n}-a_{n}\right)^{5 / 2}}{\pi^{2}}
$$

to get $(4.31)$. Thus, we set $x=\frac{a+b}{2}$ in (4.38):

$$
\frac{1}{2}\left(\int_{a}^{\frac{a+b}{2}}(\sigma-a) v(\sigma) d \sigma+\int_{\frac{a+b}{2}}^{b}(b-\sigma) v(\sigma) d \sigma\right) \geq \frac{1}{16}(b-a)^{2}(a+b) .
$$

Let be

then

$$
\theta(x)= \begin{cases}x-a & \text { if } x \in] a, \frac{a+b}{2}[, \\ b-x & \text { if } x \in] \frac{a+b}{2}, b[;\end{cases}
$$

$$
\begin{aligned}
& \int_{a}^{\frac{a+b}{2}}(\sigma-a) v(\sigma) d \sigma+\int_{\frac{a+b}{2}}^{b}(b-\sigma) v(\sigma) d \sigma=\int_{a}^{b} \theta(\sigma) v(\sigma) d \sigma \\
& \left.\leq\left(\int_{a}^{b}(\theta(\sigma))^{2} d \sigma\right)^{1 / 2}\left(\int_{a}^{b} v(\sigma)\right)^{2} d \sigma\right)^{1 / 2}=\frac{(b-a)^{3 / 2}}{2 \sqrt{3}}\left(\int_{a}^{b}(v(\sigma))^{2} d \sigma\right)^{1 / 2}
\end{aligned}
$$


Inequalities (4.40) and (4.41) give

$$
\begin{aligned}
\frac{1}{16}(b-a)^{2}(a+b) & \left.\leq \frac{1}{2 \sqrt{3}}(b-a)^{3 / 2}\left(\int_{a}^{b} v(\sigma)\right)^{2} d \sigma\right)^{1 / 2} \\
\frac{\sqrt{3}}{8}(b-a)^{1 / 2}(a+b) & \leq\left(\int_{a}^{b}(v(\sigma))^{2} d \sigma\right)^{1 / 2} \\
\frac{\sqrt{3}}{8}(b-a)^{3 / 2} & \leq\left(\int_{a}^{b}(v(\sigma))^{2} d \sigma\right)^{1 / 2} .
\end{aligned}
$$

To get (4.39) it is sufficient to have

$$
\forall n \quad \frac{\sqrt{3}}{8}\left(b_{n}-a_{n}\right)^{3 / 2} \geq 2 \varepsilon \frac{\left(b_{n}-a_{n}\right)^{5 / 2}}{\pi^{2}}, \quad \frac{\sqrt{3}}{16} \cdot \frac{1}{\pi^{2}} \frac{1}{\left(b_{n}-a_{n}\right)} \geq \varepsilon .
$$

As $0 \leq a_{n}<b_{n}<1$,

is sufficient.

$$
\frac{\sqrt{\varepsilon}}{16} \cdot \frac{1}{\pi^{2}} \geq \varepsilon
$$

Remark 4.11 (Discretized Problems). Once the discretization processes have led to stiffness matrices that are $M$-matrices, and that assumptions of Theorem 2.5 are ensured, the convergence proofs are classical.

\section{Conclusion}

The question of existence of Lagrange multipliers for optimal control of variational inequalities is not an easy one. Our analysis let us think that there are usually no Lagrange multipliers (associated with the Lagrange system we have defined) for the infinite dimensional case. So, we answer partially to the open question set in [9]. In addition we hope we have contributed to clarify the relations between all the optimality systems that have been established via different techniques. Our opinion is that the most useful one (from a numerical purpose) is the one given by Ito and Kunisch [14], which makes precise what have been done in Barbu [2].

\section{REFERENCES}

[1] D.R. Adams, S. Lenhart and J. Yong, Optimal control of variational inequalities. Appl. Math. Optim. 38 (1998) 121-140.

[2] V. Barbu, Optimal control of variational inequalities. Pitman, Boston, Res. Notes Math. 100 (1984).

[3] M. Bergounioux, Optimal control of an obstacle problem. Appl. Math. Optim. 36 (1997) 147-172.

[4] M. Bergounioux, Optimal control of problems governed by abstract variational inequalities with state constraints. SIAM J. Control Optim. 36 (1998) 273-289.

[5] M. Bergounioux, Augmented lagrangian method for distributed optimal control problems with state constraints. J. Optim. Theory Appl. 78 (1993) 493-521.

[6] M. Bergounioux and H. Dietrich, Optimal control of problems governed by obstacle type variational inequalities: A dual regularization-penalization approach. J. Convex Anal. 5 (1998) 329-351.

[7] M. Bergounioux, M. Haddou, M. Hintermueller and K. Kunisch, A comparison of interior point methods and a Moreau-Yosida based active set strategy for constrained optimal control problems. Report 98-15 Université d'Orléans (1998).

[8] M. Bergounioux and H. Zidani, Pontryagin principle for problems governed by parabolic variational inequalities. SIAM J. Control Optim. 37 (1999) 1273-1290.

[9] A. Bermudez and C. Saguez, Optimal control of variational inequalities. Optimality conditions and numerical methods. Collection Free Boundary Problems, Application and Theory, Vol. IV. Maubusson, Res. Notes Math. 121 (1984) $478-487$.

[10] A. Bermudez and C. Saguez, Optimal control of a Signorini Problem. SIAM J. Control Optim. 25 (1987) $576-582$.

[11] P.G. Ciarlet and P.A. Raviart, Maximum principle and uniform convergence for the finite element method. Comput. Methods Appl. Mech. Engrg. 2 (1973) 17-31. 
[12] W. Hackbusch, Elliptic Differential Equations, Theory and Numerical Treatment. Springer Verlag, Berlin, Ser. Comput. Math. 18 (1992).

[13] K. Ito and K. Kunisch, An augmented Lagrangian technics for variational inequalities. Appl. Math. Optim. 21 (1990) $223-241$.

[14] K. Ito and K. Kunisch, Optimal control of elliptic variational inequalities, to appear.

[15] S. Kurcyusz, On the existence and nonexistence of Lagrange multipliers in Banach spaces. J. Optim. Theory Appl. 5 (1976) $81-110$.

[16] F. Mignot, Contrôle dans les inéquations variationnelles elliptiques. J. Funct. Anal. 22 (1976) 130-185.

[17] F. Mignot and J.P. Puel, Optimal control in some variational inequalities. SIAM J. Control Optim. 22 (1984) $466-476$.

[18] F. Mignot and J.P. Puel, Contrôle optimal d'un système gouverné par une inéquation variationnelle parabolique. $C$. R. Acad. Sci. Paris Sér. I Math. 298 (1984) 277-280.

[19] D. Tiba and F. Tröltzsch, Error estimates for the discretization of state constrained convex control problems. Num. Funct. Anal. Optim. 17 (1996) 1005-1028.

[20] L. Wenbin and J.E. Rubio, Optimality conditions for strongly monotone variational inequalities. Appl. Math. Optim. 27 (1993) 291-312.

[21] J. Zowe and S. Kurcyusz, Regularity and stability for the mathematical programming problem in Banach spaces. Appl. Math. Optim. 5 (1979) 49-62. 